\title{
Model Reduction of Behavioural Systems
}

\author{
Carolyn Beck* John Doyle ${ }^{\dagger}$
}

\begin{abstract}
We consider model reduction of uncertain behavioural models. $\mathrm{Ma}$ chinery for gap-metric model reduction and multidimensional model reduction using Linear Matrix Inequalities is extended to these behavioural models. The goal is a systematic method for reducing the complexity of uncertain components in hierarchically developed models which approximates the behavior of the full-order system. This paper focuses on component model reduction that preserves stability under interconnection.
\end{abstract}

\section{Introduction}

In this paper we consider the problem of reducing uncertain be havioural systems of the type proposed by D'Andrea and Paganini [5]. The motivation for this problem comes from the desire to reduce the complexity of separate component models in a system in order to reduce the complexity of the full system. Unfortunately, the critical issue of what consitutes a good approximation of a component is problem specific and depends in detail on the rest of the system to which the component is connected, as well as on the performance requirements on that system. For example, in the standard plant/controller feed back system, approximating either the plant or controller may change a stable feedback system to an unstable one if the system is not robust to the approximation error. More generally, any approximation made to a component may result in large subsequent differences in the ful system, including instability, depending on the system to which it is connected.

While it is impossible to guarantee without further assumptions that any properties of the full system will be preserved if a component is approximated, we can add some reasonable and mild assumptions about the properties of the interconnection that will allow us to guarantee, for example, that stability will be maintained. This allows us to develop an order reduction methodology for belavioral models that is general, natural, and does not depend on detailed knowledge of the rest of the system. While it is conservative when compared to what might be possible if the entire system is considered, it has the advantage of being applied purely at the component level. Our methodology provides guaranteed upper error bounds, and maintains robust stability of interconnected systems if the error is less than a certain stability radius. It has the additional appealing interpretation of approximating component behaviors as subspaces of $\dot{L}_{2}$.

The results in this paper build on a number of results from model reduction [[7], [8], [13], [17], [18], [25], [26]], particularly balanced truncation and its recent extension to multi-dimensional (MD) and/or uncertain input-output (IO) systems with guaranteed upper bounds on the error in the $\mathcal{Q}$-norm [25]. Recently, this method has been used to determine necessary and sufficient conditions for obtaining minimal realizations of $\mathrm{MD} /$ uncertain systems in the $\mathcal{Q}$-norm [2]. Unfortunately the systems considered in these methods consist of only one component which is modelled as a linear fractional transformation (LFT) on an uncertainty/frequency structure. We extend these methods to develop model reduction techniques for interconnected behavioural system components.

After defining our notation, we give a brief review of the behavioural system framework and the gap metric in section 2 . We

\footnotetext{
"Electrical Engineering, M/S 116-81, California [ustitute of Teclinology, Pasadena, CA 91125

'Control and Dynamical Systems, M/S 116-81, Califormia lnstitute of Technology, Pasadena, CA 91125
}

then discuss model reduction and robust stability properties for general 1D behavioural systems in section 3. Relevant model reduction results for standard IO systems are reviewed in conjuction with behavioural system model reduction discussions. In section 4 , we extend these techniques to behavioural system models which contain uncertainty. We show that if uncertain behavioural system components are reduced such that the resulting error is less than a specified stability radius then the stability of the interconnected reduced system implies stability of the interconnected full system. We present one solution method to this reduction problem, via solution of a set of coupled linear matrix inequalities (LMIs) in section 5. Solutions to these LMIs may be thought of as sufficient conditions, and we discuss the several sources of potential conservativeness and possibilities for reducing them. Throughout this paper, we try to relate the concepts involved with behavioural systems to those of standard $1 \mathrm{D}$ feedback systems.

\section{Background}

We first define the notation used in this paper, and then present relevant background material in behavioural system representations and the gap metric. For a more extensive treatment of these subjects, see references [27],[1], [11],[10] and [24].

\subsection{Notation}

The notation we use is as follows. $\mathcal{H}_{2}, \mathcal{H}_{\infty}$ denote the Hardy spaces of possibly vector- or matrix-valued functions with analytic continuation on the unit disc, and $\mathcal{L}_{2}, \mathcal{L}_{\infty}$ the corresponding Lebesgue spaces of functions square integrable and essentially bounded, respectively, on the unit circle, each with norms $\|\cdot\|_{2},\|\cdot\|_{\infty} . \mathcal{R} \mathcal{H}_{\infty}$ and $\mathcal{R} \mathcal{L}_{\infty}$ are the subspaces of $\mathcal{H}_{\infty}$ and $\mathcal{L}_{\infty}$ whose elements are rational functions. We represent the integers by $\mathbf{Z}$, the time shift operator by $z^{-1}$, and the identity matrix by $I$, where the dimensions will be assumed to be clear from the context, or will otherwise be stated. The maximum singular value of $A$ is denoted $\bar{\sigma}(A)$, and $A^{*}$ denotes the adjoint.

\subsection{Behavioural System Representations}

The most striking feature of the representation of dynamical systems in the behavioural framework, as proposed by Willems [27], is the fact that there are no explicit inputs and outputs. Instead, the system is viewed as a phenomenon to be modelled, which produces elements that are referred to as outcomes. From a mathematical model for the phenomenon we call determine a set, $\mathcal{B}$, of possible outcomes, which is called the behaviour of the model. In particular, if dynamical systems are considered in this context, then the phenomenon produces outcomes which are functions of time. A dynamical system is defined in a behavioural framework as follows [27].

Definition 1 A dynamical system $\mathbf{\Sigma}$ is a triple $\boldsymbol{\Sigma}=(T, W, \mathcal{B})$, with $T \subseteq \mathbf{Z}$ the time axis, $W$ the signal space, and $\mathcal{B} \subseteq W^{T}$ the behaviour. Here, $W^{T}$ represents the set of all maps from $T$ to $W$, and $W=\mathbf{R}^{4}$.

In order to incorporate uncertainty into our models, we adapt the output nulling representation defined by Weiland [28] to describe 1D behavioural systems. A brief summary of this type of behavioural system representation follows. For more details, see [5] and [28]. We consider the equations:

$$
\begin{aligned}
& p=A x+B w \\
& 0=C x+D w
\end{aligned}
$$

where $A, B, C, D$ are constant, finite dimensional matrices and $x, w \in$ $\mathcal{L}_{2}$. The vector dimensions of $x$ and $w$ will not be specified unless pertinent to the discussion. 
The behaviour of a dynamical system $\Sigma$ is then characterized in this framework by

$$
\mathcal{B}=\left\{w \in \mathcal{L}_{2} \mid \exists x \in \mathcal{L}_{2} \text { satisfying (1) }\right\}
$$

This system has representation matrix $R=\left[\begin{array}{ll}A & B \\ C & D\end{array}\right]$, which we henceforth denote by $R=\{A, B, C, D\}$. We define a frequency/uncertainty parameter $\Delta \in \Delta$, where we assume $\Delta$ is an operator on $\mathcal{L}_{2}$ with the following structure:

$$
\Delta=\left\{\operatorname{diag}\left[\delta_{1} I_{q_{1}}, \ldots, \delta_{r} I_{q_{r}}\right], \delta_{i}: \mathcal{L}_{2} \mapsto \mathcal{L}_{2}\right\}
$$

Typically, we define $\delta_{1}=z^{-1}$ and the remaining $\delta_{i}$ as uncertainties or perturbations to the system. Uncertainty is then incorporated into our behavioural system model in a linear fractional manner by setting $x=\Delta p$. Thus, for a given $\Delta \in \Delta$, we explicitly write the LFT on $R$ and $\Delta$ as:

$$
\Delta * R=D+C \Delta(I-A \Delta)^{-1} B
$$

assuming the inverse of $(I-A \Delta)$ exists. (In [5] this LFT is denoted by $\mathcal{S}(\Delta, R)$ ). We define such a system behaviourally as follows.

Deflnition 2 An uncertain (LTI) dynamical system is a parameterized family $\left\{\Sigma_{\Delta}: \Delta \in \Delta\right\}$ of dynamic systems denoted by the quadruple $\boldsymbol{\Sigma}_{\boldsymbol{\Delta}}=\left(\mathbf{Z}, \mathbf{R}^{q}, \mathcal{B}, \Delta\right)$ whose behaviour can be expressed by

$$
\mathcal{B}=\left\{w \in \mathcal{L}_{2}:(\Delta * R) w=0 \text { for any } \Delta \in \Delta\right\}
$$

This behavioural representation is denoted $(\Delta, R)$, and is called a Generalized Output Nulling (GON) representation.

A GON representation is called regular if $D$ is surjective, dependent if $(\Delta * R)$ is not surjective $\forall \Delta \in \Delta$, and singular if it is neither regular nor dependent [5].

We want to reduce the behavioural representation matrix, $R$, with guaranteed error bounds such that if the interconnected system is stable with the reduced representation matrix, $R_{r}$, then the interconnected system is stable with the full representation matrix, $R$. Addjtionally, we would like the behaviours described by $R$ and $R_{r}$ to be close to each other.

\subsection{The Gap Metric}

The results in this paper for $1 \mathrm{D}$ behavioural systems with no uncertainty (i.e., $\Delta=z^{-1} I$ ) are essentially equivalent to existing results using the gap metric. We present a general review of the gap which includes the gap metrics of both [10] and [24], and discuss relevant robust stability properties of these metrics. We note that although existing gap metric results are developed for continuous time systems, the identical results for discrete systems also hold.

The gap may be defined between subspaces, behaviours, and IO systems using normalized coprime factors. We begin by considering the gap between two closed subspaces $\mathcal{S}_{1}$ and $\mathcal{S}_{2}$ of a Ifilbert space $\mathcal{H}$, defined as

$$
\delta_{\mathcal{H}}\left(\mathcal{S}_{1}, \mathcal{S}_{2}\right)=\left\|\Pi_{\mathcal{S}_{1}}-\Pi_{\mathcal{S}_{2}}\right\|
$$

where $\Pi_{\mathcal{S}_{1}}$ is the orthogonal projection on $\mathcal{S}_{1}$. Note that $\delta_{\mathcal{H}}\left(\mathcal{S}_{1}, \mathcal{S}_{2}\right)$ is a metric, and satisfies

$$
0 \leq \delta_{\mathcal{H}}\left(\mathcal{S}_{1}, \mathcal{S}_{2}\right) \leq 1 .
$$

For the purpose of exploring model reduction methods, we will be required only to consider problems where the gaps between subspaces are strictly less than 1 (for the general case see [10] and [24] and references therein). In this case, we can use the directed gap, defined as

$$
\vec{\delta}_{\mathcal{K}}\left(\mathcal{S}_{1}, \mathcal{S}_{2}\right)=\left\|\left(I-\Pi_{\mathcal{S}_{1}}\right) \Pi_{\mathcal{S}_{2}}\right\|=\sup _{x \in \mathcal{S}_{1},\|x\|_{2}=1}\left(\inf _{y \in \mathcal{S}_{2}}\|x-y\|_{2}\right)
$$

to derive the following alternative expression for $\delta_{\mathcal{H}}\left(\mathcal{S}_{1}, \mathcal{S}_{2}\right)<1$

$$
\delta_{\mathcal{K}}\left(\mathcal{S}_{1}, \mathcal{S}_{2}\right)=\vec{\delta}_{\mathcal{H}}\left(\mathcal{S}_{1}, \mathcal{S}_{2}\right)=\vec{\delta}_{\mathcal{H}}\left(\mathcal{S}_{2}, \mathcal{S}_{1}\right) \text {. }
$$

In particular, consider the typical plant/controller feedback configuration. The plant and controller are modelled as LFTs on the fre quency structure $\Delta=z^{-1} I$, with resulting transfer functions denoted by $P_{\mathrm{i}}$ and $C$. As in [10] and [24], we denote a nornalized right coprime factorization ( $r C f)$ of $P_{i}$ by $\left[N_{i}, M_{i}\right]$, and a normalized left coprime factorization $\left(l c f\right.$ by $\left[\tilde{N}_{i}, \tilde{M}_{i}\right]$. Similarly, $\left[N_{c}, M_{c}\right]$ and $\left[\tilde{N}_{c}, \tilde{M}_{c}\right]$ will denote normalized right and left coprime factorizations of a controller $C$. We write

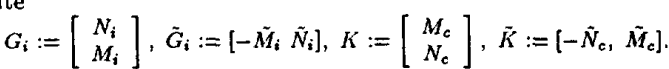

Recall that $\left[\tilde{N}_{i}, \tilde{M}_{i}\right]$ is a normalized $l c f$ of $P_{i}$ if, and only if, i) $P_{i}=\bar{M}_{i}^{-1} \tilde{N}_{i}$, ii) $\tilde{G}_{i} \in \mathcal{H}_{\infty}$, iii) there exists an $Y \in \mathcal{K}_{\infty}: \tilde{G}_{i} Y=I$ (the coprimeness condition) and iv) $\tilde{G}_{i} \tilde{G}_{i}^{*}=I$ (the normalization condition). Similar conditions can be given for normalized refs. Such factorizations always exist, with $\operatorname{deg}\left(G_{i}\right)=\operatorname{deg}\left(\tilde{G}_{i}\right)=\operatorname{deg}\left(P_{i}\right)$. Note that $\tilde{G}_{i} G_{i}=\tilde{K} K=0$, thus $\left[\begin{array}{ll}G_{i} & \tilde{G}_{i}^{*}\end{array}\right]$ and $\left[\begin{array}{cc}K & \tilde{K}^{*}\end{array}\right]$ are unitary.

The $\mathcal{H}_{2}$-graph $\mathcal{G}_{i}$ of $P_{i}$ is the closed subspace of $\mathcal{H}_{2}$ consisting of all pairs $w=(u, y)$ such that $y-P_{i} u=0$ or

$$
\mathcal{G}_{i}=G_{i} \mathcal{H}_{2}=\left\{w \in \mathcal{H}_{2}: \tilde{G}_{i} w=0\right\}
$$

The $\mathcal{H}_{2}$-gap between two systems, $P_{1}$ and $P_{2}$, is defined accordingly as the distance between their respective $\mathcal{H}_{2}$-graphs, $\mathcal{G}_{1}$ and $\mathcal{G}_{3}$. The $\mathcal{L}_{2}$-gap is defined similarly. The formula for the $\mathcal{H}_{2}$-gap metric derived in [11] is

$$
\delta_{\mathcal{H}_{2}}\left(P_{1}, P_{2}\right)=\vec{\delta}_{\mathcal{H}_{3}}\left(\mathcal{G}_{1}, \mathcal{G}_{2}\right)=\inf _{Q \in \mathcal{H}_{\infty}}\left\|G_{1}-G_{2} Q\right\|_{\infty}=\inf _{Q \in \mathcal{H}_{\infty}}\left\|\left[\begin{array}{c}
G_{2}^{*} G_{1}-Q \\
\tilde{G}_{2} G_{1}
\end{array}\right]\right\|_{\infty}
$$

For details see [10] and the references therein.

Of particular relevance to the problem we consider is the $\nu$-gap, defined by Vinnicombe [24]. Vinnicombe defines the $\nu$-gap in such a way that, provided $\delta_{\nu}\left(P_{1}, P_{2}\right)<1$, it is equal to the $\mathcal{L}_{2}$-gap and is defined as

$$
\delta_{\nu}\left(P_{1}, P_{2}\right)=\delta_{\mathcal{L}_{2}}\left(\mathcal{G}_{1}, \mathcal{G}_{2}\right)=\left\|\tilde{G}_{2} G_{1}\right\|_{\infty}
$$

The last expression follows from the definition of $\vec{\delta}_{\mathcal{L}_{2}}\left(\mathcal{G}_{1}, \mathcal{G}_{2}\right)$ and the

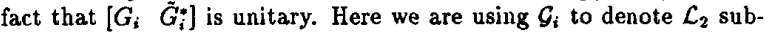
spaces.

The assumption in this paper that all of the gaps are strictly less than 1 is justified by the fact that we obtain approximations to $\tilde{G}_{1}$ by a lower order $\tilde{G}_{2}$ that satisfies $\left\|\tilde{G}_{1}-\tilde{G}_{2}\right\|_{\infty}<\epsilon<1$. Since it is easily shown that

$$
\delta_{\nu}\left(P_{1}, P_{2}\right) \leq \delta_{\boldsymbol{R}_{2}}\left(P_{1}, P_{2}\right) \leq\left\|\tilde{G}_{1}-\tilde{G}_{2}\right\|_{\infty}
$$

we may always assume that all gaps are strictly less than 1 . This greatly simplifies the discussion without incurring any loss of generality.

\subsection{Approximation in the Gap}

As in [15], [10], and [24], for a plant $P$ and controller $C$ connected in a standard feedback configuration, we consider the matrix transfer function

$$
\Pi_{P, C}=\left[\begin{array}{l}
I \\
P
\end{array}\right](I-C P)^{-1}[I C],
$$

and define the associated generalized stability maryin as $b_{P, C}=$ $\left\|\Pi_{P, C}\right\|_{\infty}^{-1}$ if $\Pi_{P, C}$ is stable, and $b_{P, C}=0$ otherwise. Using the normalized $r c f$ and $l c f$ representations in $(6), \Pi_{P, C}=G(\tilde{K} G)^{-1} \tilde{K}$, thus,

$$
b_{P, C}=\left\|(\tilde{K} G)^{-1}\right\|_{\infty}^{-1} .
$$

For this problem setup, both the $\mathcal{H}_{2}$-gap and the $\nu$-gap have the following property $([10],[24])$ :

Property 1:

Given a nominal plant $P_{3}$ and a controller $C$ then:

$\Pi_{P_{2}, C}$ is stable for all plants, $P_{2}$, satisfying $\delta\left(P_{1}, P_{2}\right) \leq \beta$ if, and only if, $\beta<b_{P_{1}, c}$.

This property tells us that any plant at a distance less than $\beta$ from the nominal will be stabilized by any controller stabilizing the nominal plant with a stability margin of $\beta$. The $\nu$-gap also has the additional property:

Property 2:

Given a nominal plant $P_{1}$, a perturbed plant $P_{3}$, and a number $\beta<b_{\text {opt }}(P)$ then:

$\Pi_{P_{2}, C}$ is stable for all controllers, $C$, satisfying $\beta<b_{P_{1}, C}$ if, and only if, $\delta_{\nu}\left(P_{1}, P_{2}\right) \leq \beta$, where $b_{\text {opt }}(P):=\sup _{C} b_{P, C}$, the optimal stability radius.

This second property says that any plant at a distance greater than $\beta$ from the nominal will be destabilized by some controller which stabilizes the nominal with a stability margin of at least $\beta$. Additionally, it can be shown that the performance change in replacing plant $P_{0}$ by $P_{1}$ is bounded by $\delta_{v}\left(P_{0}, P_{1}\right)$. These properties make $\delta_{v}(\cdot$.$) def.$ initely most excellent, and ideal for a priori model reduction of the plant, since we need only assume that the controller eventually used will have reasonable generalized stabjlity margin. 
We briefly summarize balanced model reduction methods and state resulting upper error bounds. Consider the standard IO system described by an LFT on the frequency structure $\Delta=z^{-1} I$ and $R=\{A, B, C, D\}$. This system is considered balanced if there exists a diagonal matrix $\Sigma=\operatorname{diag}\left[\sigma_{1} I_{1}, \sigma_{2} I_{2}, \ldots, \sigma_{n} I_{n}\right]$ with $\sigma_{1}>\sigma_{2}>\cdots>\sigma_{n} \geq 0$ such that $A \Sigma A^{*}-\Sigma+B B^{*}=0$ and $A^{*} \Sigma A-\Sigma+C^{*} C=0$. The entries $\sigma_{i}$, of $\Sigma$, are called the Hankel singular values of the system, and $I_{i}$ are used to indicate that the multiplicity of $\sigma_{i}$ may be greater than 1 . Assuming $R$ is balanced, partition $R$ and $\Sigma$ into the following subblocks:

$$
R=\left[\begin{array}{ccc}
A_{r} & A_{12} & B_{r} \\
A_{21} & A_{22} & B_{2} \\
C_{r} & C_{2} & D
\end{array}\right] \quad \Sigma=\left[\begin{array}{cc}
\Sigma_{r} & 0 \\
0 & \Sigma_{2}
\end{array}\right]
$$

$\Sigma_{r}=\operatorname{diag}\left[\sigma_{1} I_{1}, \cdots, \sigma_{k} I_{k}\right]>0$ and $\Sigma_{2}=\operatorname{diag}\left[\sigma_{k+1} I_{k+1}, \cdots, \sigma_{n} I_{n}\right] \geq 0$ Then truncate $R$ to $R_{r}=\left\{A_{r}, B_{r}, C_{r}, D\right\}$. Bounds for the error resulting from this truncation as derived in [7], [8], and [25] are

$$
\sigma_{k+1} \leq\left\|\Delta * R-\Delta_{r} * R_{r}\right\|_{\infty} \leq 2 \sum_{i=k+1}^{n} \sigma_{i} .
$$

For a system represented by a normalized $r c f, G$, a balanced truncation reduction method with an upper error bound in the graph metric has been computed by Meyer [17]. Balancing and truncating the $\mathrm{rcf}$ representation $G$ results in a reduced, norinalized $r c f, G_{r}$ with

$$
\delta\left(P, P_{r}\right)=\inf _{Q}\left\|G-G_{r} Q\right\|_{\infty} \leq 2 \sum_{i=k+1}^{n} \sigma_{i}
$$

whese $\delta(\cdot, \cdot)$ denotes the graph metric and $\sigma_{i}$ are the Hankel singular values of the representation for $G$. The graph metric and the gap metric are closely related, [11], and bounds for approximation in the gap metric have also been computed [12]. Additionally, we note that Weiland develops a more general notion of balanced representations in the behavioural framework, along with an upper bound for balanced truncation which is equivalent to that in (8) [28].

In view of the properties of the $\delta_{\nu}(\cdot, \cdot)$ we would like to model reduce using this as our metric, but currently no such method with associated bounds exists. It is widely accepted among the cognoscenti that our best option is to use

$$
\delta_{\nu}\left(P_{0}, P_{1}\right) \leq\left\|\tilde{G}_{0}-\tilde{G}_{1}\right\|_{\infty}
$$

and make the norm on the right small using IIankel norm approximation or balanced truncation [9]. See [24] for more details.

\section{Model Reduction of 1D Behavioural Sys- tems}

In order to more readily connect results in the gap metric and $1 \mathrm{D}$ behavioural system representations in anticipation of generalizing to include uncertainty, we will henceforth denote $\hat{G}=(\Delta * R)$, and $\tilde{K}=(\Delta * F)$, where $R$ and $F$ are representation matrices for components in an interconnected behavioural system and $\Delta$ is the frequency/uncertainty set for the system. Note that we abuse the notation here, as $(\Delta * R)$ is not meant to represent the system at one specific $\Delta$, but instead represents the system as an operator and $\Delta$ represents the set $\Delta$. This notation is used throughout this paper. We assume $R$ and $F$ are regular representations. Given one component, $\mathcal{G} \subset \mathcal{L}_{2}$, of an interconnected behavioural system, where $\mathcal{G}$ is represented by $(\Delta, R)$, we want to approximate $\mathcal{G}$ by a simpler representation. One natural approach is to seek approximations to the behavior itself as a subspace in $\mathcal{L}_{2}$, which would be similar to approximations in the $\mathcal{L}_{2}$ gap or $\nu$-gap. We cannot approximate these subspaces directly, but we can determine approximation bounds using error bounds on $\tilde{G}$ as suggested by the $\nu$-gap properties. As in the gap theory, approximation of behaviours can be related directly to properties of interconnections.

Consider the behavioural system described by the interconnection of two behavioural components defined as the subspaces $\mathcal{G}$ and $\mathcal{K}$ of $\mathcal{L}_{2}$. We adopt much of the gap notation but do not assume that $\mathcal{G}$ and $\mathcal{K}$ are the graphs of IO operators. Assume we have normalized transfer functions $G$ and $\tilde{G}$ such that

$$
\mathcal{G}=G \mathcal{L}_{2}=\left\{w \in \mathcal{L}_{2}: \tilde{G} w=0\right\}
$$

and $\left[\begin{array}{ll}G & \tilde{G}^{*}\end{array}\right]$ is unitary. If we consider normalized behavioural descriptions, robust stability criteria are easily constructed which are analogous to robustness in the gap metric. We construct these criteria in such a manner that they reduce to the robust stability criteria in the gap metric given in [10] for the standard feedback configuration with components $P$ and $C$. The general definition for normalized behavioural representations we use throughout this paper is as follows: Definition $3 A$ behavioural representation $(\Delta * R)$ is normalized if

$$
(\Delta * R)(\Delta * R)^{*}=I
$$

In the $1 \mathrm{D}$ case we also can find $G$ such that $\left[G \tilde{G}^{*}\right]$ is unitary. We choose the term normalized to correspond with the IO definition of systems represented by normalized coprime factors. This concept is the same as that of coisometric defined by Weiland [28]. Weiland shows that, as in the standard case, if we are given a behavioural representation matrix $R=\{A, B, C, D\}$ which is not normalized, we can compute an equivalent normalized representation by solving an algebraic Ricatti equation (Theorem 3.26, [28]), which is equivalent to the normalization methods developed for solving $\mathcal{H}_{\infty}$ optimal control problems in [6].

The interconnected system we will consider is

$$
\tilde{G} w=0 ; \tilde{K} v=0
$$

$$
w+v=n
$$

where $n$ represents noise injected at the interconnection. We will as sume that a well-formed interconnection involves the maps from $n$ to $w$ and $v$ being bounded. Basically, as we don't know in detail how our component will be connected, it is reasonable to expect that if noise is injected at the interconnection, then this noise will not be greatly amplified. In the standard feedback configuration considered in the gap case, this has a clear interpretation as $\left\|\Pi_{p, C}\right\|_{\infty}$. The implications of this assumption are less clear in the behavioural case. For example, it excludes interconnections that yield singular representations. Nevertheless, we make this assumption on interconnections as a reasonable starting point.

\subsection{Reduction of Behavioural Components}

The behavioural system representation allows us to perform model reduction and robustness analysis for more general system descriptions than the standard IO setup. Consider the interconnected behavioural system described by (10). We form the input/output relations

$$
w=\Pi_{\dot{G}, \dot{k}} n, v=\Pi_{\dot{K}, \dot{G}} n
$$

quite readily by computing

$$
\Pi_{\tilde{G}, \tilde{K}}=\left[\begin{array}{c}
\tilde{G} \\
\tilde{K}
\end{array}\right]^{-1}\left[\begin{array}{c}
0 \\
\tilde{K}
\end{array}\right] \text { and } \Pi_{\tilde{K}, \tilde{G}}=\left[\begin{array}{c}
\tilde{G} \\
\tilde{K}
\end{array}\right]^{-1}\left[\begin{array}{c}
\tilde{G} \\
0
\end{array}\right]
$$

Note that in the standard feedback setting $\left\|I_{\tilde{Q}, \tilde{K}}\right\|_{\infty}^{-1}=b_{P, C}$. We assume the above inverses exist and are stable, in which case we say the intercomnected behavioural system is nominally stable. Additionally, this assumption implies that $\tilde{G}$, as a matrix, has more columns than rows and is therefore guaranteed to have a kernel, but $\tilde{G}^{*}$ may not.

Directly applying the balanced truncation model reduction method previously described to $R=\{A, B, C, D\}$ results in a reduced representation matrix $R_{r}=\left\{A_{r}, B_{r}, C_{r}, D_{r}\right\}$ and corresponding $\Delta_{r}$ such that $\left\|\tilde{G}-\tilde{G}_{r}\right\|_{\infty}$ is guaranteed to be bounded by some value, say $\epsilon$. Our first objective is to state conditions under which behavioural systems are robust to such reductions, so we first consider the behavioural system described by (10) with additive uncertainty. That is, suppose $\tilde{G}$ is perturbed to $\tilde{G}+\Delta_{1}$ where $\Delta_{1} \in \Delta_{1}$. While we are most interested in the case where $\Delta_{\mathrm{t}}$ represents approximation error, it is also possible that $\Delta_{1}$ represents unstructured uncertainty which is possibly time-varying. The following robust stability lemmas for behavioural system representations are trivial extensions of corresponding gap results, but are stated for the purpose of generalization to uncertain behavioural systems. A sketch of the proof for Lemma 1 is given, as the same metlod of proof can be used in the uncertain case.

Lemma 1 Suppose the interconnected behavioural system described by (10) is nominally stable, and $\Delta_{G} \in \Delta_{G}$ with $\left\|\Delta_{G}\right\| \leq \epsilon$, then the corresponding perturbed system

$$
\left(\tilde{G}+\Delta_{G}\right) w=0 ; \tilde{K} v=0
$$


is stable for all $\left\|\Delta_{G}\right\| \leq \epsilon$ iff

$$
\left\|\left[\begin{array}{l}
\bar{G} \\
\tilde{K}
\end{array}\right]^{-1}\left[\begin{array}{l}
I \\
0
\end{array}\right]\right\|<\frac{1}{\epsilon} .
$$

Proof:

Substituting $v=n-w$, our uncertain behavioural system interconnection equations can be written in matrix form as

$$
\left(\left[\begin{array}{c}
\bar{G} \\
\bar{K}
\end{array}\right]+\left[\begin{array}{c}
\Delta_{G} \\
0
\end{array}\right]\right) w=\left[\begin{array}{c}
0 \\
\bar{K}
\end{array}\right] n
$$

Rewriting this in a transfer function form from $n$ to $w$ gives

$$
w=\left(I+\left[\begin{array}{c}
\tilde{G} \\
\tilde{K}
\end{array}\right]^{-1}\left[\begin{array}{c}
\Delta_{c} \\
0
\end{array}\right]\right)^{-1}\left[\begin{array}{c}
\tilde{G} \\
\tilde{K}
\end{array}\right]^{-1}\left[\begin{array}{c}
0 \\
\tilde{K}
\end{array}\right] n
$$

Thus, stability is guaranteed iff

$$
\left\|\left[\begin{array}{c}
\tilde{G} \\
\tilde{K}
\end{array}\right]^{-1}\left[\begin{array}{l}
I \\
0
\end{array}\right]\right\|<\frac{1}{\epsilon}
$$

We can then prove the following theorem.

Theorem 1 Suppose $\left(\Delta_{1} * R_{1}\right)=\tilde{G}_{1}$ is normalized and $\left\|\Pi_{\tilde{G}_{1}, \hat{K}}\right\|_{\infty}<\frac{1}{\epsilon}$. If the behavioural system given by (10) with $\tilde{G}_{1}$ is stable, then it is stable when $\tilde{G}_{1}$ is replaced by any $\tilde{G}_{2}$ with the property

$$
\left\|\tilde{G}_{1}-\tilde{G}_{2}\right\|_{\infty} \leq \epsilon
$$

In particular, suppose $\tilde{G}_{2}$ represents the nominal system and $\tilde{G}_{1}$ the reduced system. Then we can normalize $\tilde{G}_{2}$ and truncate using Meyer's algorithm, giving us a normalized $\bar{G}_{1}$. We then want to find the smallest dimension $\tilde{G}_{1}$ satsfying $\left\|\tilde{G}_{1}-\tilde{G}_{2}\right\|_{\infty} \leq\left\|\Pi_{\tilde{G}_{1}, \tilde{K}}\right\|_{\infty}^{-1}$, to satisfy the assumptions of Theorem 1 . This theorem follows innmediately from Lemma 1 and the following Lemma, the proof of which is very straightforward and therefore not presented here.

Lemma 2 Suppose $\tilde{G}$ is normalized and $\left\{\Pi_{2} \|<\frac{1}{\epsilon}\right.$, then

$$
\left\|\left[\begin{array}{c}
\tilde{G} \\
\tilde{H}
\end{array}\right]^{-1}\left[\begin{array}{l}
I \\
0
\end{array}\right]\right\|<\frac{1}{\epsilon}
$$

The above theorem can be compared to similar theorems using the $\nu$-gap. It gives sufficient conditions for safely reducing a component model, but is potentially conservative. A less conservative theorem, which follows immediately from Vinnicombe's results [24], is

Theorem 2 Suppose $\tilde{G}_{1}$ is normalized and $\left\|\Pi_{\tilde{G}_{1}, \dot{k}}\right\|_{\infty}<\frac{1}{6}$. If the behavioural system given by (10) with $\tilde{G}_{1}$ is stable, then it is stable when $\tilde{G}_{1}$ is replaced by any $\tilde{G}_{2}$ with the property $\left\|\tilde{G}_{1}-\tilde{G}_{2}\right\|_{\infty}<1$ and $\delta_{\mathcal{L}_{2}}\left(\mathcal{G}_{1}, \mathcal{G}_{2}\right)<\epsilon$

The test $\delta_{\mathcal{L}_{2}}\left(\mathcal{G}_{1}, \mathcal{G}_{2}\right)<\epsilon$ is the least conservative possible if the only information given about the intercomnection is that $\left\|\Pi_{\tilde{G}_{1}, \tilde{K}}\right\|_{\infty}<$ 1. Unfortunately we can't use $\delta_{\mathcal{L}_{3}}\left(\mathcal{G}_{1}, \mathcal{G}_{2}\right)$ directly, so we will have to be content with model reduction based on the bound $\delta_{\mathcal{L}_{2}}\left(\mathcal{G}_{1}, \mathcal{G}_{2}\right) \leq$ $\left\|\tilde{G}_{1}-\tilde{G}_{2}\right\|_{\infty}$. This approach appears to be effective in the gap case, and can be extended in the next section to behavioral systems with uncertainty.

\section{Behavioural Systems with Uncertainty}

In an attempt to develop a model reduction method for uncertain behavioural systems, we first consider balanced truncation model reduction for uncertain 10 systems. We would like to extend this method to behavioural systems described by a representation matrix $R=\{A, B, C, D\}$ and frequency/uncertainty structure $\Delta$ as given in (2). We present a set of sufficient conditions on the error resulting from reducing uncertain behavioural realizations which, if satisfied, guarantee stability of the resulting interconnected system.

To determine the robustness of behavioural system stability to model reduction, we must have some measure of the error incurred by such a reduction, and a precise notion of stability for such a system. We utilize the following definitions of $\mathcal{Q}$-stability, and the $\mathcal{Q}$-norm for this purpose.
Definition 4 The uncertain system represented by $(R, \Delta)$ is said to be $\mathcal{Q}$-stable if there exists a non-singular matrix $T$ such that $T \Delta=\Delta T$ $\forall \Delta$ and

$$
\bar{\sigma}\left(T A T^{-1}\right)<1 \text {. }
$$

Analogous to the definition of $\mathcal{Q}$-stability of uncertain behavioural systems, we define the following $\mathcal{Q}$-norm by which to measure the model reduction error.

Definition 5 The $\mathcal{Q}$-norm of a system representation $(R, \Delta)$ is given by

$$
\|\Delta * R\|_{Q}=\inf \left\{\gamma: \exists T \text { such that } \bar{\sigma}\left(\left[\begin{array}{cc}
T A T^{-1} & \frac{1}{\gamma^{\frac{T}{\gamma}}} T B \\
\frac{1}{\gamma^{\frac{1}{2}} C T^{-1}} & \frac{1}{\gamma} D
\end{array}\right]\right)<1\right\}
$$

where $T \Delta=\Delta T$.

For 1D systems, the $\mathcal{Q}$-norm is the same as the $H_{\infty}$ norm. For uncertain systems, $Q$-stability and performance are necessary and sufficient for robust stability and performance, when $\Delta$ represents arbitrary linear operators. For the repeated $\delta$ case considered here, the proof of this involves a generalization [22] that directly combines existing results [16], [23], [21]. For input-output systems, the importance of stability and robustness is clear and we will use $\mathcal{Q}$-stability and performance for our final interconnected systems. This allows our $\Delta$ to be time-varying (and even nonlinear) but would be conservative if $\Delta$ had additional structure, such as time-invariance.

For behavioural representations, the use of a stable representation matrix $R$ is perhaps less fundamental but is very convenient for manipulation and computation. Stable $R$ generalizes the use of stable coprime factor representations for input-output systems and then norms can be used to define generalizations of normalized coprime factors. These generalizations allow us to compare robust stability results formulated in the gap netric and provide for a natural representation and measure of error in the behavioural framework.

\subsection{Model Reduction Results for Uncertain Systems}

A brief review of model reduction results for MD/uncertain IO systerns is presented. The reader is referred to [25] and [2] for full details. We again consider a system represented as an LFT on a $\Delta$ structure. In this case the frequency/uncertainty set $\Delta$ is defined as in (2). Generally, one $\delta_{i}$ represents the system frequency variable, e.g., $z^{-1}$, and the remaining $\delta_{i}$ are arbitrary operators on $\mathcal{L}_{2}$ representing uncertainty. If all $\delta_{i}$ represent frequency variables, reduction corresponds to state order reduction, as in the $1 \mathrm{D}$ case. If $\delta_{i}$ represent uncertainty, reduction corresponds to simplification of the uncertainty descriptions.

As in the preceeding discussions, we consider only similarity transformations which commute with the $\Delta$ structure, to which we refer as allowable transformations. Thus, an allowable transformation $T$ has block-diagonal structure.

The definition of balanced we use differs slightly from the standard definition. Non-strict inequalities are used rather than equalities in the Lyapunov equations as allowable solutions to the equalities may not exist for uncertain systems.

Definition 6 : $A$ rcalizalion $\{A, B, C, D\}$ is balanced if $\exists \Sigma=\operatorname{diag}\left[\sigma_{1} I_{1}, \sigma_{2} I_{2}, \ldots, \sigma_{n} I_{n}\right]>0$ such that

$$
A \Sigma A^{*}-\Sigma+B B^{*} \leq 0 \text { and } A^{*} \Sigma A-\Sigma+C^{*} C \leq 0
$$

We discuss reduction of a $2 D$ system for notational convenience. The stated results hold for any number of blocks. We assume the system representation,

$$
R=\left[\begin{array}{ccc}
A_{11} & A_{12} & B_{1} \\
A_{21} & A_{22} & B_{2} \\
C_{1} & C_{2} & D
\end{array}\right] \Delta=\left\{\operatorname{diag}\left[\delta_{1} I_{1}, \delta_{2} I_{2}\right]: \delta_{i} \in \mathcal{C}\right\}
$$

is balanced, $\mathcal{Q}$-stable, and is partitioned, along with $\Sigma$, as follows:

$$
\begin{gathered}
A_{i j}=\left[\begin{array}{cc}
A_{i j}, & A_{i j_{12}} \\
A_{i j_{21}} & A_{i j_{23}}
\end{array}\right] \quad B_{i}=\left[\begin{array}{c}
B_{i r} \\
B_{i,}
\end{array}\right] C_{i}=\left[\begin{array}{ll}
C_{i,} & C_{i,}
\end{array}\right] \\
\Sigma=\operatorname{diag}\left[\Sigma_{i_{r}}, \Sigma_{1_{2}}, \Sigma_{2 r}, \Sigma_{2_{2}}\right] \\
\Sigma_{i_{r}}=\operatorname{diag}\left[\sigma_{i 1} I_{i 1}, \ldots, \sigma_{i k_{i}} I_{i k_{i}}\right]>0 \text { and } \\
\Sigma_{i_{3}}=\operatorname{diag}\left[\sigma_{i\left(k_{i}+1\right)} I_{i\left(k_{1}+1\right)}, \ldots, \sigma_{i n_{i}} I_{i n_{1}}\right]>0
\end{gathered}
$$

where $\sigma_{i 1} \geq \sigma_{i 2} \geq \cdots \geq \sigma_{i n_{i}}$.

The balanced truncation model reduction theorem is as follows: 
Theorem 3 If we truncate $R$ to $R_{r}=\left[\begin{array}{ccc}A_{11,} & A_{12 r} & B_{1 r} \\ A_{21,} & A_{22 r} & B_{2 r} \\ C_{1,} & C_{2 r} & D\end{array}\right]$ (and $\Delta$ to $\Delta_{r}$ with corresponding partitions), then $R_{r}$ is balanced, $\Delta_{r} * R_{r}$ is Q-stable, and

$$
\left\|(\Delta * R)-\left(\Delta_{r} * R_{r}\right)\right\|_{Q} \leq 2 \sum_{i=1}^{2} \sum_{j=k_{1}+1}^{n_{i}} \sigma_{i j}
$$

Thus, if $\sum_{i=1}^{2} \sum_{j=k_{i}+1}^{n_{i}} \sigma_{i j}$ is sinall, then we can reduce the system order and the resulting error in the $Q$-norm of the system is small. In the $1 \mathrm{D}$ case previously discussed, if this error is less than $\left\|\Pi_{2}\right\|^{-1}$, then the reduced component can be used in the interconnected system without causing instability. This robust stability condition generalizes to the uncertain case.

In addition to the reduction theory presented, necessary and sufficient conditions for exact reducibility of uncertain systems in the $\mathcal{Q}$-norm have recently been found [2] which are also applicable to behavioural system representations. We summarize these results, without proof, in the following. Proofs are given in [2].

Theorem 4 Let $R=\{A, B, C, D\}$ be a $\mathcal{Q}$-stable system representation matrix with uncertainty/frequency structure $\Delta \in \Delta$, with $\operatorname{dim}(\Delta)=n$. Then there exists $\left(\Delta_{r}, R_{r}\right)$, with $\operatorname{dim}\left(\Delta_{r}\right)=k<n$, such that $\left\|\left(\Delta * R-\Delta_{r} * R_{r}\right)\right\|_{Q} \leq \epsilon$ if, and only if, there exist allowable $X \geq 0$ and $Y \geq 0$ satisfying

(i) $A Y A^{*}-Y+B B^{*} \leq 0$

(ii) $A^{*} X A-X+C^{*} C \leq 0$

(iii) $X Y \geq \epsilon^{2} I$, with the lowest $n-k$ eigenvalues of $X Y$ being equal to $\epsilon^{2}$

where $\epsilon \geq 0$.

For $\epsilon>0$, the proof of Theorem 4 follows directly from application of machinery developed by Packard [20]. The exact reducibility case, that is, the $\epsilon=0$ case, is more involved. In proving sufficiency in Theorem 4 for $\epsilon=0$, we actually construct a reduced system realization, $R_{r}$, by balancing and trurcating $R$. Alternatively, in proving necessity for $\epsilon=0, R_{r}$ may be any system matrix as long as it is of smaller dimension. Additionally, $\mathcal{Q}$-stability of $G$ is not required for the necessity proof. We can apply Theorem 4 to uncertain behavioural system representations in the same way that we apply the basic model reduction results.

\subsection{Robust Stability of Interconnections}

Obviously, we would like to maintain as many similarities as possible between the MD/uncertain case and the $1 D$ case. In particular, we would like the uncertain behavioural system representations to be normalized. However, in the uncertain case, there is no guarantee that an allowable solution to the required Ricatti equations exists which yields a normalized realization. In fact, such a solution most likely does not exist. Thus, rather than attempting to find a normalized realization, we utilize the following concepts of expansiveness and contractiveness. Definition 7 The behavioural system representation, $(\Delta, R)$, is $\alpha$ contractive (expansive) if, for all $\Delta \in \Delta$ such that $\Delta^{*} \Delta=I$,

$$
(\Delta * R)(\Delta * R)^{*} \leq(\geq) \alpha^{2} I
$$

If $\alpha=1$ then we simplify the terminology to just contractive or expansive. Equivalently, a representation $(\Delta, R)$ is $\alpha$-contractive for all unitary $\Delta$ if

$$
\text { there exists allowable } T: \bar{\sigma}\left(\left[\begin{array}{cc}
T A T^{-1} & \frac{1}{\alpha^{\frac{1}{3}}} T B \\
\frac{1}{\alpha^{\frac{1}{2}} C T^{-1}} & \frac{1}{\alpha} D
\end{array}\right]\right) \leq I
$$

and is $\alpha$-expansive for all unitary $\Delta$ if

$$
\text { there exists allowable } T: \underline{\sigma}\left(\left[\begin{array}{cc}
T A T^{-1} & \frac{1}{\sigma^{\frac{1}{3}}} T B \\
\frac{1}{a^{\frac{1}{2}}} C T^{-1} & \frac{1}{\alpha} D
\end{array}\right]\right) \geq I
$$

where $g(\cdot)$ refers to the smallest non-zero singular value.

Note that in the above definitions, we evaluate expansiveness and contractiveness only for unitary $\Delta$. While in general this gives only a subset of the behaviors, it will include those that are worst-case for stability and performance of the interconnected system, because for any $\mathcal{L}_{2}$ stability or performance condition, unitary $\Delta$ are always worstcase for operator $\Delta$. This is not true, of course, for $\Delta$ that include real parametric uncertainty, which is not considered in this paper.
We require that the realization we reduce be contractive and $\alpha$ expansive (for $\alpha<1$ ), in order that Lemmas 1 and 2, and Theorem 1 generalize to the uncertain case with as little additional conservativeness in the stability margins as possible. This is discussed in more detail following the statements of Lemma 3 and Theorem 5.

The result and proof of Lemma 1 hold for the uncertain case exactly as stated, using the $\mathcal{Q}$-norm for the system matrices and the appropriate operator norm for $\Delta_{1}$. As in the $1 \mathrm{D}$ case, Theorem 5 follows immediately from Lemmas 1 and 3 .

Theorem 5 Suppose $\left(\Delta_{1}, R_{1}\right)$ is $\alpha$-expansive with $\alpha<1$ and $\left\|\Pi_{R_{1}, F}\right\|_{Q}<\frac{1}{1}$. If the behavioural system given by (10) with $\left(\Delta_{1}, R_{1}\right)$ is stable, then it is stable when $\left(\Delta_{1}, R_{1}\right)$ is replaced by any $\left(\Delta_{2}, R_{2}\right)$ with the property

$$
\left\|\Delta_{1} * R_{1}-\Delta_{2} * R_{2}\right\|_{Q} \leq \alpha \epsilon
$$

Lemma 3 Suppose $(R, \Delta)$ is $\alpha$-expansive with $0<\alpha<1$ and $\left\|\Pi_{R, F}\right\|_{Q}<\frac{1}{\epsilon}$, then

$$
\left\|\left[\begin{array}{c}
\Delta * R \\
\Delta * F
\end{array}\right]^{-1}\left[\begin{array}{l}
I \\
0
\end{array}\right]\right\|_{0}<\frac{1}{\alpha \epsilon}
$$

The proof of Lemma 3 relies on the showing that the following inequalities hold.

$\left\|\left[\begin{array}{c}\Delta * R \\ \Delta * F\end{array}\right]^{-1}\left[\begin{array}{l}I \\ 0\end{array}\right]\right\|=\left\|\Pi_{R, F}(\Delta * R)^{*}\left\{(\Delta * R)(\Delta * R)^{*}\right\}^{-1}\right\| \leq \frac{1}{\alpha}\left\|\Pi_{R_{1} F}\right\|$

The relations between the norms above provide some insight as to why we want our realizations $(\Delta, R)$ to be contractive, and $\alpha<1$ as close to 1 as possible. Given contractiveness we can show

$$
\left\|\left[\begin{array}{l}
\Delta * R \\
\Delta * F
\end{array}\right]^{-1}\left[\begin{array}{l}
I \\
0
\end{array}\right]\right\|_{Q} \geq\left\|\mathrm{II}_{R, F}\right\|_{Q}
$$

That is, we can determine a lower bound which provides information on the conservativeness of the allowed model reduction error.

\section{LMI Solutions for Behavioural Model Re- duction}

Using the robust stability results formulated for behavioural systems in the preceeding section, and the model reduction teclniques discussed in section 4.1, we arrive at a set of coupled LM1 conditions which, if satisfied, provide a robustly stable model reduction method for the behavioural system framework. These LMI conditions are derived by determining if either the system representation we are given, or an equivalent system representation, satisfies a set of norm bounds and $\mathcal{Q}$ stability requirements. We first discuss equivalence of different system representations for behavioural systems.

\subsection{Equivalent System Representations}

To apply the model reduction techniques and robust stability analysis previously described, stability and $\alpha$-expansiveness (with $\alpha<1$ ) of representations, $(\Delta, R)$, are required. Additionally, we want this representation to be contractive. If the given representation matrix, $R$ is not stable, $\alpha$-expansive and contractive, we determine if an equivalent representation exists which does satisfy these constraints, where we use the following notion of equivalence:

Definition 8 Given a representation $(\Delta, R)$ for a behavioural system, an equivalent representation is defined by any representation matrix $\hat{R}$ such that for each $\Delta \in \Delta$

$$
\{w:(\Delta * R) w=0\} \equiv\{\hat{w}:(\Delta * \hat{R}) \hat{w}=0\}
$$

Predictably, transforming the system matrices of $R$ by an allowable similarity transformation results in an equivalent representation, as does multiplying the system matrices $C$ and $D$ on the left by a constant nonsingular matrix. Less obvious is the use of what is known as output injection in the standard 10 case, that is, using a constant matrix, $L$, to construct a new representation $R=\{(A+L C),(B+L D), C, D\}$. Recall that using an output nulling representation implies that the output is always 0 , thus, adding $L \cdot 0=L C x(k)+L D w$ to $p$ does not affect the behaviour, resulting in an equivalent representation.

For 1D systems representations, all equivalent representations are obtained by output injection, similarity transformations and truncations. Whether we obtain all equivalent MD/uncertain system representations by these same methods remains a topic of current research. 
Thus the class of equivalent systems we consider may be somewhat restricted, introducing another source of conservativeness into our solutions.

\subsection{LMI Problem Formulation}

Following the $1 \mathrm{D}$ case, we want to find an equivalent representation, $\hat{R}$, for $R$, that is contractive and $\alpha$-expansive (with $\alpha<1$ as close to 1 as possible), which we then balance and truncate. Note that if $\hat{R}$ is contractive, we know there exists an allowable transformation such that the Lyapunov inequalities required for balanced truncation are satisfied, allowing us to eliminate the $Q$-stability constraint on $\hat{R}$.

To formulate the coupled LMI conditions required to find such a $\hat{R}$, we first write the above constraints using the definitions in (13) and (14) for contractiveness and expansiveness. Given the representation matrix, $R$, and the associated $\Delta$ structure, we want to determine if there exist constant matrices $L$ and (nonsingular) $Z$, such that $\{(A+L C),(B+L D), Z C, Z D\}$ is contractive and $\alpha$-expansive. Thus, we must determine if there exist $L, Z$ and allowable similarity transformations $T_{1}$ and $T_{2}$ satisfying:

(i) $\left[\begin{array}{cc}T_{1} & 0 \\ 0 & I\end{array}\right]^{-1} R^{*}\left[\begin{array}{ll}I & L \\ 0 & Z\end{array}\right]^{*}\left[\begin{array}{cc}T_{1} & 0 \\ 0 & I\end{array}\right]^{2}\left[\begin{array}{ll}I & L \\ 0 & Z\end{array}\right] R\left[\begin{array}{cc}T_{1} & 0 \\ 0 & I\end{array}\right]^{-1} \leq I$

(ii) $\left[\begin{array}{cc}T_{2} & 0 \\ 0 & I\end{array}\right]^{-1}\left[\begin{array}{ll}I & L \\ 0 & Z\end{array}\right] R_{\alpha}\left[\begin{array}{cc}T_{2} & 0 \\ 0 & I\end{array}\right]^{2} R_{\alpha}^{*}\left[\begin{array}{ll}I & L \\ 0 & Z\end{array}\right]^{*}\left[\begin{array}{cc}T_{2} & 0 \\ 0 & I\end{array}\right]^{-1} \geq I$ where $R=\{A, B, C, D\}$ and $R_{\alpha}=\left\{A, \frac{1}{\alpha^{\frac{1}{3}}} B, \frac{1}{\alpha^{\frac{1}{2}}} C, \frac{1}{\alpha} D\right\}$. We then pre and post-multiply (i) by $\left[\begin{array}{cc}T_{1} & 0 \\ 0 & I\end{array}\right]$ and make the following matrix variable substitutions, $X_{1}=T_{1}^{2}, \quad X_{2}=T_{1}^{2} L$ and $X_{3}=L^{*} T_{1}^{2} L+Z^{*} Z$, after which we can reformulate (i) in the following LMI form.

Problem Statement 1a Find $X$ satisfying:

$$
\begin{aligned}
& \text { 1. } X=\left[\begin{array}{ll}
X_{1} & X_{2} \\
X_{2}^{*} & X_{3}
\end{array}\right]=X^{*}>0 \quad X_{1} \Delta=\Delta X_{1} \\
& \text { 2. } R^{*} X R-\left[\begin{array}{ll}
I & 0 \\
0 & 0
\end{array}\right] X\left[\begin{array}{ll}
I & 0 \\
0 & 0
\end{array}\right]-\left[\begin{array}{ll}
0 & 0 \\
0 & I
\end{array}\right] \leq 0
\end{aligned}
$$

To put (ii) into LMI form, we pre and post-multiply by both $\left[\begin{array}{cc}T_{2} & 0 \\ 0 & I\end{array}\right]$ and $\left[\begin{array}{cc}I & L \\ 0 & Z\end{array}\right]^{-1}$ and make the following substitutions

$$
T=T_{2}^{2} \text { and } V=\left[\begin{array}{c}
-L \\
I
\end{array}\right]\left(Z^{*} Z\right)^{-1}\left[-L^{*} I\right]
$$

Using the solution $X$ from Problem 2a, solutions $T$ and $\alpha$ satisfying (ii) may then be determined by computing $L=X_{1}^{-1} X_{2},\left(Z^{*} Z\right)=$ $X_{3}-X_{2} X_{1}^{-1} X_{2}^{*}$ and $V$, and solving the following LMIs.

Problem Statement $1 \mathrm{~b}$ Find $T$ and the maximum $\alpha<1$ satisfying:

$$
\text { 1. } T=T^{*}>0 ; T \Delta=\Delta T
$$

$$
\text { 2. } R_{\alpha}\left[\begin{array}{ll}
T & 0 \\
0 & I
\end{array}\right] R_{\alpha}^{*}-\left[\begin{array}{cc}
T & 0 \\
0 & 0
\end{array}\right]-V \geq 0
$$

For LMI problems such as those given by Problem Statements 1a and $1 \mathrm{~b}$, applications of interior point methods for convex optimization problems have given quite promising results. See [3], [4], [14] and the references therein for more details. Additional applicable results have been obtained on minimization of the maximum eigenvalue of a symmetric matrix [19].

We want to find a matrix $X$ satisfying $2 a$ which results in the largest possible $\alpha<1$ in $2 \mathrm{~b}$, therefore giving the least conservative bounds. However, determining the solution for which $\alpha$ is maximized over both $X$ and $T$ requires an algorithm which iterates over both parts $a$ and $b$ of Problem 1. Unfortunately, this coupled LMI problem is not convex as stated here, thus the LMI solutions discussed in the above references are not directly applicable. Alternative algorithms for optimizing this coupled LMI problem are currently being explored.

To find a reduced representation, $R_{r}$, for $R$, with which to apply Theorem 5 , we first balance and truncate $\hat{R}$. Unfortunately, in the $\mathrm{MD} /$ uncertain case, balancing and truncating does not preserve contractiveness or $\alpha$-expansiveness (with the same $\alpha$ ). We can, use the error from truncating to bound the difference in contractiveness and expansiveness from the original representation to the reduced representation. We can also apply the method presented in Problem 1 to $R_{r}$ to find an equivalent $\hat{R}_{\tau}$ which is contractive and $\alpha_{r}$-expansive, again with $\alpha_{r}<1$ and as close to 1 as possible, in order to get the least conservative bounds for Theorem 5 . As in the $1 \mathrm{D}$ case, we want to find the smallest dimension $R_{r}$ satisfying $\left\|\Delta * R-\Delta_{r} * R_{r}\right\|_{Q}<\alpha_{r}\left\|\Pi_{R_{r}, F}\right\|_{Q}^{-1}$. This solution method is potentially very conservative as the bounds obtained are sufficient at each of the above stages for both the full and the reduced system representations. We would eventually like to find a solution method in which equivalent contractive and $\alpha$-expansive representations are found and the balancing and truncating completed in one step.

\section{Acknowledgements}

This paper could not have been completed without the many valuable explanations and insights of Alexandre Megretskii, Keith Glover, Malcolm Smith and Glenn Vinnicombe, for which the authors are very grateful.

\section{References}

[1] Antoulos, A.C., and Willems, J., "A Behavioural Approach to Linear Exact Modeling", to appear in IEEE AC, Dec. 1993

[2] Beck, C., Doyle, J., and Glover, K., "Reducibilty of Uncertain Systems", reported in preparation, 1993

[3] Boyd, S., and El Gliaoui, L., "Method of Centers for Minimizing Generalized Eigenvalues", Linear Algebra and it's Applications, 1993

[4] Boyd, S., El Glıaoui, L., Feron, E., and Balakrishlinall, V., Linear Matrix Inequalities in System and Control Theory, to be published by Springer-Verlag

[5] D'Aundrea, R., and Paganini, F., "literconnection of Uncertain Behavioural Systems for Robust Control", $C D C$, San Antonio, TX, 1993

[6] Doyle, J., Lecture Notes, Honeywell/ONR Workshop, 1984, Minneapolis, MN.

[7] Enns, D.F., 1984, Model Reduction for Control System Design, Ph.D. Dissertation, Stanford University.

[8] Glover, K., "All optimal Hankel-norm approximations of linear multivariable systems and their $L^{\infty}$-error bounds", $I J C$, Vol.39:1115-1193, 1984

[9] Glover, K., 1993, speculation.

[10] Georgiou, T., and Smith, M.C., "Optimal Robustuess in the Gap Metric", IEEE TAC, Vol. 35 , No. 6, 673-685., 1990

[11] Georgiou, T., "On the computation of the gap metric", System Control Letlers, Vol. 11:253-257, 1988

[12] Georgiou, T., and Smit]l, M.C., "Upper and Lower Bounds for Approximation in the Gap Metric", $C D C$, Brighton, Eugland, 1991

[13] Hinrichsen, D. and Pritchard, A.J., "An improved error estimate for reducedorder models of discrete-time system", IEEE TAC, AC-35:317-320, 1990

[14] Jarre, F., "An Interior-Point Method for Minimizing the Maximum Eigenvalue of a Linear Combination of Matrices", Teclnical Report SOL 91-8, Stanford, California.

[15] McFarlane, D.C., and Glover, K., Robust Conlroller Design Using Normalized Coprime Factor Plant Descriptions, Springer-Verlag, Lecture Notes in Control and Information Sciences, 138, 1989

[16] Megretskii, A., "Necessary and Suflicient Conditions of Stability: A Multiloop Generalization of the Circle Criterion", IEEE TAC, Vol. 38, No. 5, 1993

[17] Meyer, D.G., "A Fractional Approach to Model Reduction", $A C C$, Atlanta, GA, 1988

[18] B.C. Moore, "Principal component analysis of linear systems: Controllability, observability and model reduction", IEEE TAC, Vol. 26, 1981

[19] Overton, M.L., "Large-Scałe Oplimization of Eigenvalues", New York UniverOverton, M.L., "Large-Scale Optimization of Eigenvalues",
sity, Department of Computer Science Report No. 505. 1990

[20] Packard, A., “Notes for LFT Optimal Control", University of California, Berkeley, Departinent of Mechanical Engineering Report, 1992

[21] Packard, A., and Doyle, J., "The Complex Structured Singular Value", Automatica, Vol.29, No. 1, 1993

[22] Rantzer, A., and Doyle, J., supposedly in preparation

[23] Shamma, J., "Robust Stability with Time-Varying Structured Uncertainty", to appear IEEE TAC

[24] Vinnicombe, G., "Frequency Domain Uncertainty and the Graph Topology", IEEE TAC, Vol. 38, No. 9, 1993

[25] Wang, W., Doyle, J., Beck, C., and Glover, K., "Model Reduction of LFT Systems", $C D C$, Brigliton, England, 1991

[26] Wang, W. and Safonov, M.G., "A relative error bound for discrete-time balanced stochastic truncalion", $A C C$, San Diego, CA, 1990

[27] Willems, J., "Paradigıns and Puzzles in the Theory of Dynamical Systems", IEEE TAC, Vol. 36, No. 3, 259-294, 1991

[28] Weiland, S., Theory of Approxinsation and Disturbance Attenuation for Linear Systems, PhD, University of Groningen, 1991 